\title{
The structure and poloidal dynamics of blob filaments in TJ-K
}

\author{
S Garland ${ }^{1}$, G Fuchert ${ }^{2}$, M Ramisch ${ }^{1}$, T Hirth ${ }^{1}$ \\ ${ }^{1}$ Institute of Interfacial Process Engineering and Plasma Technology, University \\ of Stuttgart, 70569 Stuttgart, Germany \\ 2 Max Planck Institute for Plasma Physics, Boltzmannstr. 2, 85748 Garching, \\ Germany \\ E-mail: garland@igvp.uni-stuttgart.de
}

\begin{abstract}
Relatively dense, field-aligned, filament-like structures (blobs) have been observed to propagate radially and poloidally through the SOL in magnetically confined fusion plasmas, and contribute significantly to SOL transport. A detailed understanding of blob structure and dynamics, and their dependence on magnetic field geometry, is of high importance in magnetic confinement physics for the prediction of heat loads on reactor wall facing components, as well as for understanding plasma confinement and neutral particle recycling. Experimentally deduced centre of mass poloidal blob velocity components, obtained using the conditional averaging technique, have been compared to an analytical blob model which has been simplified to express blob velocity in terms of the magnetic field curvature vector. Background flows are not incorporated into the analytical model, and must be added in to obtain good agreement with the experimental data. In addition, the 3D structure of blobs in TJ-K has been investigated using the conditional average of density fluctuations in two toroidally separated poloidal planes. Blobs are observed to be aligned to a flux tube near to the last closed flux surface, in the blob birth region. However at positions further along the blob trajectory, the structures do not deform according to the magnetic shear, rather they remain rigid, and retain their original form.
\end{abstract}

Submitted to: Plasma Phys. Control. Fusion 


\section{Introduction}

The density fluctuations measured in the scrape-off layer (SOL) of most magnetic confinement devices exhibit positive skewness, which often indicates the presence of mesoscale structures called blobs [1, 2]. Blobs are filamentary structures, elongated along the magnetic field lines, and have a higher pressure than the background SOL plasma. The name blob stems from their elliptical cross sectional shape. These structures are generated near the separatrix and are commonly observed to propagate radially and poloidally through the SOL towards the vacuum vessel wall, transporting heat and particles along with them. They can therefore impact equilibrium density profiles and pose a potential risk to plasma facing components [3], as well as being a potential mechanism for neutral particle recycling [4]. Therefore, the prediction of blob trajectories and transport, and the heat loads they cause on the first wall is of high importance for the design of future reactors.

In this contribution, the link between magnetic field geometry and blob properties is investigated in the TJ-K stellarator [5]. An analytical model for the geodesic curvature drive of poloidal blob velocity is compared to experimental poloidal blob velocities. In addition, 3D blob structure is determined at different positions along a blob trajectory in the SOL, with different magnetic field properties, and the effect of magnetic shear on filament structure is investigated.

Many studies have previously been carried out into blob velocity in tokamaks and stellarators, as well as other fusion research devices (e.g. [6 16]). The review paper [2] contains an overview of the measurements done in different devices. Although poloidal blob velocities are regularly measured in experiments (e.g. [11, 17 19]), their detailed study is often made difficult due to the need to accurately measure background $E \times B$ flows, in contrast to the radial velocity component, and the poloidal blob velocity is often reported to be of the order of the background flow (e.g. [8]). The driving force of blob propagation can be associated with the magnetic field line curvature, $\boldsymbol{\kappa}$, which causes cross-field blob polarization due to a curvature drift and subsequent propagation of the blob filament due to the $E \times B$ drift. An analytical blob model [20 22], treating a blob filament as an electrical circuit, connects the blob velocity to the magnetic field curvature.

The model starts from an expression of charge conservation $(\nabla \cdot \mathbf{j}=\mathbf{0})$ inside a blob filament in a cold plasma $\left(T_{\mathrm{i}}=0\right)$, which is separated from the background SOL plasma. Following reference [20], this can be written as

$$
\frac{m_{\mathrm{i}}}{B^{2}} \nabla \cdot\left(n \frac{\mathrm{d}}{\mathrm{d} t} \nabla_{\perp} \phi\right)=\nabla_{\|} j_{\|}+\frac{2}{B} \hat{\boldsymbol{b}} \cdot \boldsymbol{\kappa} \times \nabla p,
$$

where $m_{\mathrm{i}}$ is the ion mass and $B$ is the magnetic field. The plasma potential and density are $n$ and $\phi$ respectively. The term on the left hand side of the equation 
describes the current due to the polarisation drift. The right hand side contains a parallel current term and the curvature drive term, where $\nabla p$ is the plasma pressure gradient and $\hat{\boldsymbol{b}}=\boldsymbol{B} /|B|$. In previous studies on TJ-K, it has been found that collisions with neutrals have a negligible effect on blob dynamics [13], and so they are not included in the model here. In addition, parallel blob currents previously measured in TJ-K, have been found to have a relatively small impact on blob velocity in the framework of equation 1, and for this reason they are neglected in the present contribution. These assumptions correspond to the so-called inertial regime, which has been found to well suit the description of radial blob velocity scaling in TJ-K [13]. In this regime the polarisation of filaments due to the curvature term is balanced only by the polarisation current. Equation 1 is thus reduced to an expression relating the current induced by the blob curvature drive to the polarisation current, which acts to reduce the internal blob electric field,

$$
-\frac{n m_{\mathrm{i}}}{B^{2}} \frac{\mathrm{d}}{\mathrm{d} t}(\nabla \cdot \boldsymbol{E})=\frac{2}{B} \hat{\boldsymbol{b}} \cdot \boldsymbol{\kappa} \times \nabla p .
$$

Here the Boussinesque approximation has been also used as discussed in [22]. Now, by assuming solutions of the form $f=f_{0} \exp ^{i(k r-\omega t)}$ for the electric field and pressure variations, the expression becomes

$$
-\frac{n m_{\mathrm{i}} \gamma}{B^{2}} \boldsymbol{k} \cdot \boldsymbol{E}=\frac{2 p}{B} \hat{\boldsymbol{b}} \cdot \boldsymbol{\kappa} \times \boldsymbol{k}
$$

where $\gamma=\sqrt{2} c_{\mathrm{s}} / \sqrt{R \delta_{\mathrm{b}}}$ is the growth rate of the ideal interchange instability (see [23, 24]). This equation can then be rearranged to express the blob electric field in terms of the magnetic curvature,

$$
\boldsymbol{E}=-\frac{2 T_{\mathrm{e}}}{m_{\mathrm{i}}} \frac{\tilde{n}}{n} \gamma^{-1}(\boldsymbol{B} \times \boldsymbol{\kappa}),
$$

where the blob is assumed to have the temperature of the background plasma, i.e. $p=\tilde{n} T_{\mathrm{e}}$. Finally, by inserting the expression for the internal dipole blob electric field, $\boldsymbol{E}$ (assumed entirely perpendicular to $\boldsymbol{B}$ ), into the equation for the $E \times B$ drift velocity, $\boldsymbol{v}_{\mathrm{E} \times \mathrm{B}}=(\boldsymbol{E} \times \boldsymbol{B}) / B^{2}$, and using the definitions $c_{\mathrm{s}}=\left(T_{\mathrm{e}} / m_{\mathrm{i}}\right)^{1 / 2}$ and $\hat{n}=\tilde{n} / n$, the equation relating the blob velocity vector, $\boldsymbol{v}_{\mathrm{b}}$, to the magnetic curvature vector in the inertial approximation can be obtained,

$$
\boldsymbol{v}_{\mathrm{b}}=-2 c_{\mathrm{s}}^{2} \gamma^{-1} \hat{n} \boldsymbol{\kappa} .
$$

In the context of the TJ-K experiment, it is useful to express the blob velocity in terms of the field-aligned normal and geodesic magnetic curvature components, $\kappa_{\mathrm{n}}$ and $\kappa_{\mathrm{g}}$ respectively. The normal curvature is defined as the projection of the curvature vector onto a normal, $\boldsymbol{n}$, to the flux surface, and the geodesic 
curvature vector is the projection of the curvature onto the vector $\boldsymbol{g}=\hat{\boldsymbol{b}} \times \boldsymbol{n}$ (binormal direction). In this paper, it is assumed that the surface normal, $\boldsymbol{n}$, points approximately in the measured radial direction, and that the bi-normal direction, $\boldsymbol{g}$, points approximately in the measured poloidal direction (where the measuring plane lies in a circular cross-section of the torus, see figure 1). This allows the radial and poloidal velocity components to be expressed as

$$
\begin{aligned}
& v_{\mathrm{r}, \mathrm{b}}=-2 c_{\mathrm{s}}^{2} \gamma^{-1} \hat{n} \kappa_{n}, \\
& v_{\theta, \mathrm{b}}=-2 c_{\mathrm{s}}^{2} \gamma^{-1} \hat{n} \kappa_{\mathrm{g}} .
\end{aligned}
$$

Blob filaments are often said to be field-aligned, and it is therefore thought that they should become deformed in regions of high magnetic shear [25], such as close to

the X-point of a plasma with divertor geometry [24], where the magnetic field lines fan out. There are relatively few experimental investigations of the influence of magnetic shear on blob structure (examples of studies are [26, 27]). The accurate knowledge of the magnetic field geometry in TJ-K, which can be calculated using a field line tracing code, allows relatively precise measurements of filament field-alignment in different magnetic shear regions of the TJ-K SOL.

This paper begins with an introduction to the experiment TJ-K and the diagnostics used at TJ-K in section 2, following which, in section 3, the data analysis techniques applied to obtain the results are explained. The results in section 4 are split into two parts. Firstly, experimental results showing the link between the geodesic magnetic field line curvature and the poloidal dynamics of blobs are presented. After this, a second investigation into the 3D structure of blobs as they propagate through the SOL is shown. Finally the conclusions drawn from these two studies will be summarised in section 5 .

\section{Experimental setup}

Experiments were conducted in the small-sized torsatron TJ-K [5], which has a major radius of $0.6 \mathrm{~m}$ and a minor plasma radius of $0.1 \mathrm{~m}$. Electron densities are of the order of $10^{17} \mathrm{~m}^{-3}$ whilst electron temperatures are typically around $10 \mathrm{eV}$ and those of ions $1 \mathrm{eV}$, allowing Langmuir probe access to the entire plasma volume. Measurements can therefore be taken with the necessary spatio-temporal resolution to study plasma turbulence in detail. Dimensionless parameters relevant to edge plasma turbulence are similar in TJ-K to those typically found in the edge region of reactor grade experiments [5].

Magnetic fields of approximately $70 \mathrm{mT}$ are typically used, and plasma heating is carried out with up to $3 \mathrm{~kW}$ of $2.45 \mathrm{GHz}$ microwave radiation. The gases $\mathrm{H}, \mathrm{D}, \mathrm{He}$, 


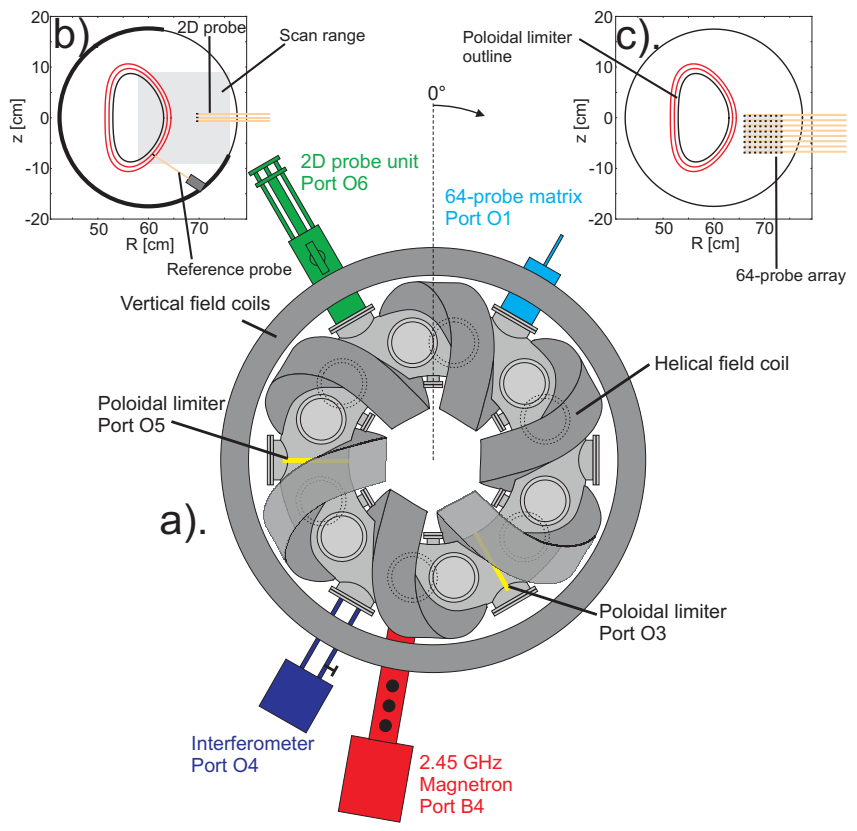

Figure 1. Top-view schematic of the TJ-K torsatron (a). The two probe diagnostics, 2D scanning probe (b) and the 64-probe matrix (c), which are separated by $60^{\circ}$, are depicted in relation to the flux surface configuration, shown in cross section in the two inset plots.

Ne and Ar are available, allowing exploration of a large parameter space relevant to turbulence.

A range of Langmuir probe diagnostics are available for use at TJ-K, with a data acquisition frequency of up to $1 \mathrm{MHz}$. For the present contribution two probe diagnostics were used: a 2D scanning probe unit, capable of sampling fluctuations within a poloidal cross section; a probe matrix comprising a square $8 \times 8$ grid of probes spaced equally by $1 \mathrm{~cm}$, and covering $49 \mathrm{~cm}^{2}$. A step size of $1 \mathrm{~cm}$ was also used for the data obtained with the scanning probe. The conditional averaging technique (see section 3) can be used on the measurements from these diagnostics in order to obtain 2D and 3D information on blobs. In typical TJ-K discharges, fluctuations in the ion saturation current, $\tilde{I}_{\mathrm{i}, \mathrm{sat}}$, can be assumed proportional to those of density due to the lack of temperature fluctuations [28]. A schematic of the TJ-K torsatron along with the diagnostics used is shown in figure 1.

In addition to the standard Langmuir probes, used to measure $I_{\mathrm{i}, \mathrm{sat}}$ and $\phi_{\mathrm{fl}}$, ohmically heated emissive probes were used to estimate the plasma potential, $\phi_{\mathrm{pl}}$. The emissive probes used were constructed in a similar way to those in [28]. By mounting the probe on the $2 \mathrm{D}$ scanning probe unit, it can be used to calculate radial electric fields, and thereby arrive at the poloidal $E \times B$ drift which is of high 
importance when studying the poloidal blob dynamics (see section 44).

In order to create a controlled environment in which to study blobs, two poloidal limiters were installed at the toroidal angles $\phi=150^{\circ}$ and $270^{\circ}$, giving an enlarged SOL as well as an extended region of approximately uniform magnetic field line connection length. The last closed flux surface in such a configuration is defined by a flux surface shape cut into a smooth metal disk, blocking the poloidal cross section (see figure 1). In contrast to previous blob experiments at TJ-K, these limiters were constructed for a magnetic flux surface configuration which gives a substantially enlarged SOL on the outboard side at the outer measuring ports, with a maximum physical SOL width of approximately $14 \mathrm{~cm}$ at the outer mid-plane, allowing more detailed study of blob filaments passing through regions with different magnetic field properties.

\section{Data analysis}

In order to obtain poloidal blob velocities, 2D information on the density fluctuations is needed. To obtain this, individual time series measured with the $2 \mathrm{D}$ scanning probe at discrete positions in the poloidal cross section were conditionally averaged using a fixed reference probe to provide the trigger conditions. The trigger condition for blob detection was taken to be fulfilled when the ion saturation current signal, $\tilde{I}_{\mathrm{i} \text {,sat }}$, rose from below the detection threshold of two times the standard deviation, $2 \sigma_{\mathrm{I}_{\mathrm{i}, \mathrm{sat}}}$, to above this threshold in the space of one time step. A sample $\tilde{I}_{\mathrm{i}, \text { sat }}$ signal measured by the reference probe is shown in figure $2 \mathrm{a}$ ). The choice of blob detection threshold was made so that high amplitude fluctuations were triggered upon, and not those corresponding to the background turbulent fluctuations. Plot c) of figure 2 shows a histogram of the entire $0.5 \mathrm{~s}$ ion saturation current signal, a portion of which is plotted in plot a). Here the trigger condition can be seen to lie in the non-Gaussian tail of the distribution.

It has been observed in TJ-K that blobs have a preferred generation region [29], making placement of the reference probe easier, as well as analysis of the subsequent blob trajectories. The reference probe was placed close to the LCFS, in the region where the majority of blobs have been observed to occur. The method of conditional averaging requires a simultaneous reference probe signal for every signal obtained using the scanning probe. The reference probe signals are searched for instances where the trigger condition is fulfilled, $t_{\text {trig }}$, which occurs typically many hundreds of times per $0.5 \mathrm{~s}$ time series. Sub-windows of equal length, centred on $t_{\text {trig }}$, are then extracted from the scanning probe signal and the mean of these sub-windows is determined. The process is repeated for all the measuring points in the $2 \mathrm{D}$ poloidal cross section. The $2 \mathrm{D}$ density fluctuations resulting from this technique show the 

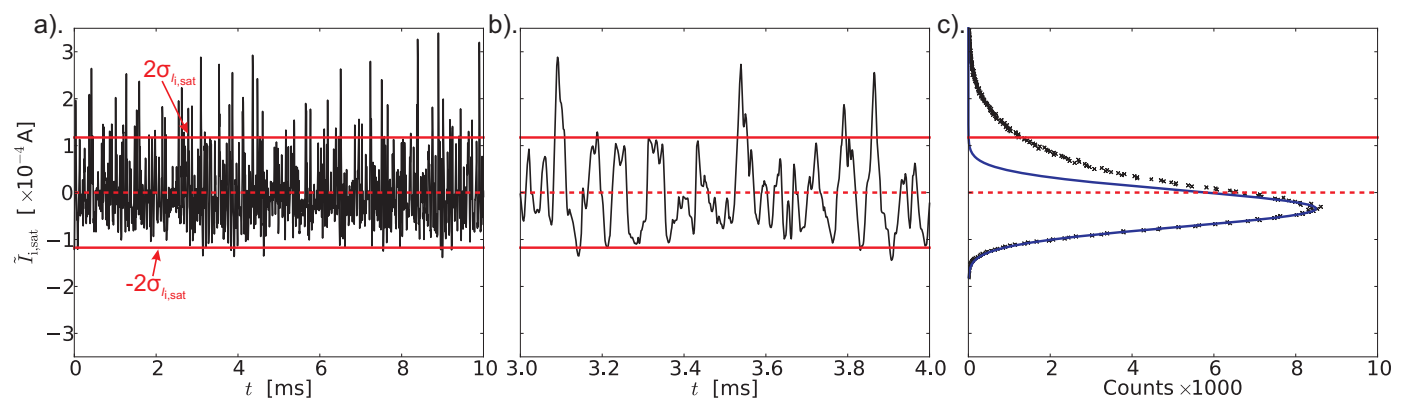

Figure 2. a). A sample $10 \mathrm{~ms}$ time series of $\tilde{I}_{\mathrm{i} \text { sat }}$ taken by the reference probe during a He discharge. The $2 \sigma_{I_{\mathrm{i}, \mathrm{sat}}}$ threshold is shown in red. b). The same time series zoomed in on a $1 \mathrm{~ms}$ interval. c). A histogram of the entire $0.5 \mathrm{~s}$ signal (crosses) with a gaussian curve overplotted.

average blob dynamics in the torus cross section for coherent structures which fulfil the trigger condition at the reference probe position.

Figure 3 shows an example of three frames from the conditional average of $\tilde{I}_{\mathrm{i} \text {,sat }}$. A blob can be seen to propagate from near the last closed flux surface in the radial and poloidal directions towards the vacuum vessel walls. Velocity components can be determined from the displacement of blob centres of mass (CoMs) between successive frames. An average blob velocity is obtained by determining the mean velocity within a restricted range of the SOL over which the blob's poloidal velocity is approximately constant. This range varies between shots, however it is typically of the order of $20 \mu \mathrm{s}$.

The auto and cross-conditional averages (ACA and CCA respectively) at 2 positions along a blob CoM trajectory, obtained from conditional averaging, are shown in figure 4. The same reference probe was used for both CCAs, and the ACAs have been shifted in time to allow comparison with the CCAs. The reduction in peak amplitude of the CCA between plots 4a) and b), indicates that not all the fulfilled trigger conditions at the reference probe result in a large amplitude fluctuation at a constant $\Delta t$ further out the SOL, probably due in part to a spread in blob velocity. This hypothesis is supported by the observed broadening of the CCA peak further along the trajectory, since blobs with different speeds arrive at different time delays, $\Delta t$, in the conditional average. The peak broadening in the cross-conditional average combined with a reduction in the peak amplitude could result in an overestimation of blob sizes, $\delta_{\mathrm{b}}$, as well as an underestimate of the $\hat{n}$ parameter at positions further away from the reference probe. The maximum overall effect on the model velocity estimate (equation (7) is of the order of $5 \%$, when the initial values of $\delta_{\mathrm{b}}$ and $\hat{n}$ are taken at the reference probe as opposed to the average values over a restricted portion of the blob trajectory. This effect is intrinsic to the conditional average technique 


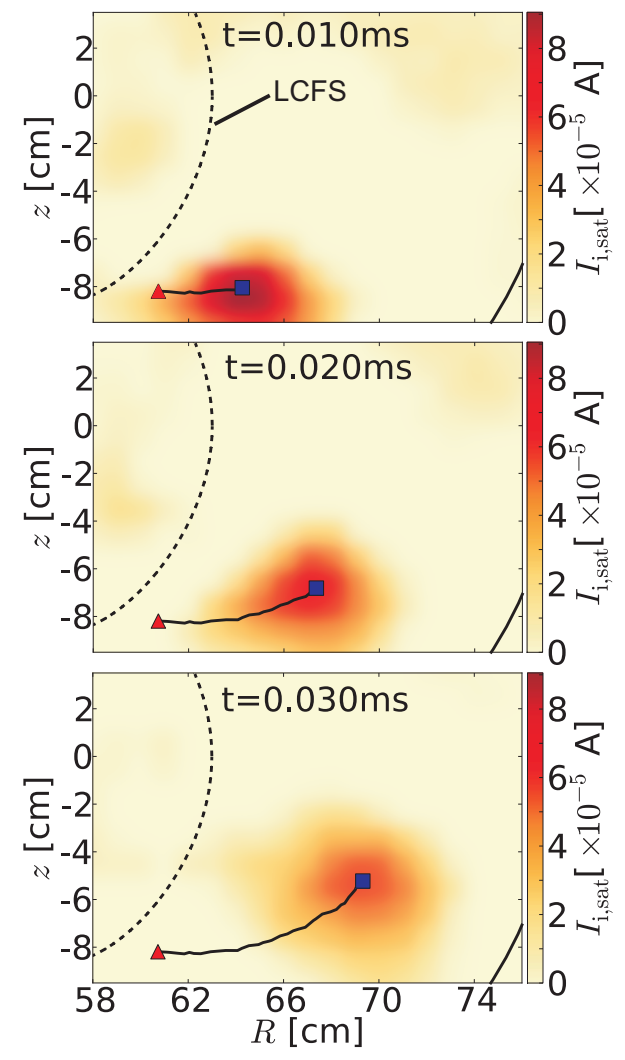

Figure 3. Three frames of the conditionally averaged $I_{\mathrm{i}, \text { sat }}$ fluctuations (higher density fluctuations are shown in red). A blob can be seen propagating radially and poloidally over a period of $20 \mu$ s, and the trajectory has been traced with a black line from the reference probe position (red triangle) to the blob CoM (blue square). The mid-plane of the torus is at $z=0$.

and is difficult to compensate for because of the difficulty in determining whether the reduced peak amplitude is due to a spread in blob velocities or in fact due to some blobs decreasing in amplitude before reaching positions further out in the SOL.

Another potential pitfall when using the conditional average technique is the averaging together of structures ejected simultaneously into the SOL from different poloidal angles. This scenario is unlikely in TJ-K due to a previous study where the link between drift waves in the edge region and blobs in the SOL was made clear [13]. In this paper the distribution of waiting times between events for drift wave and blobs was determined for a hydrogen and a helium discharge, giving peaks at time delays of approximately 90 and $76 \mu$ s respectively. Taking the case of helium, a most commom delay time of $76 \mu \mathrm{s}$ is much larger than the blob lifetime, defined by the width at $\tilde{I}_{\mathrm{i} \text { sat }}=\max \left(\tilde{I}_{\mathrm{i}, \mathrm{sat}}\right) / \mathrm{e}$, determined from figure 4 as $18 \mu \mathrm{s}$, which means the probability of two blobs overlapping each other is relatively low. 

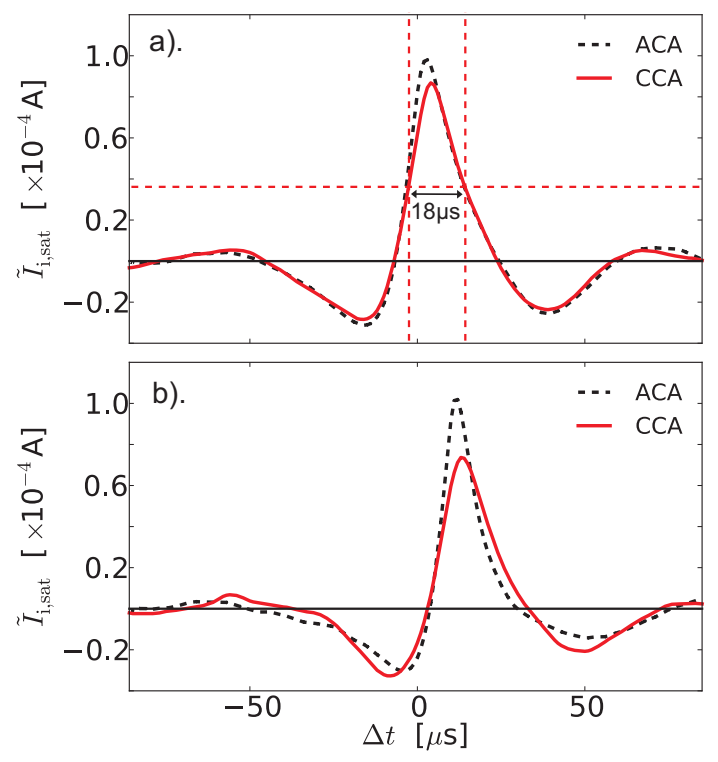

Figure 4. The auto and cross-conditional averages at two positions along the CoM trajectory of the conditionally averaged blob, with plot a) near the reference probe and plot b) around $15 \mu$ s later.

The information required to study the 3D structure of blob filaments can also be obtained by using the conditional average technique, on simultaneous measurements at two toroidally separated measuring positions, using the 2D scanning probe and the 64-probe matrix (the probe matrix can obtain data simultaneously with all 64 probes). The conditional average carried out using a common, reference probe then results in the average blob shape and dynamics simultaneously at the two toroidally separated measuring positions. The effect of an increased lifetime of the blob due to a spread in blob speeds is avoided by choosing a reference probe located in the probe matrix, allowing the blob structure to be analysed at close to $\Delta t=0$.

\section{Results}

\subsection{Geodesic curvature drive of $v_{\theta}$}

Since the analytical model discussed in section 1 treats blobs as separate from the background plasma, in order to understand the geodesic curvature drive of blob propagation in the poloidal direction it was necessary to also determine the $E \times B$ poloidal background flow. The poloidal flow velocity was determined from the equation $v_{\mathrm{E}_{\mathrm{r}} \times \mathrm{B}}=E_{\mathrm{r}} / B$. Radial electric field, $E_{\mathrm{r}}$, measurements were achieved by calculating the gradient of directly measured plasma potential, measured using ohmically heated emissive probes mounted on the 2D scanning unit. Figure 5 shows 


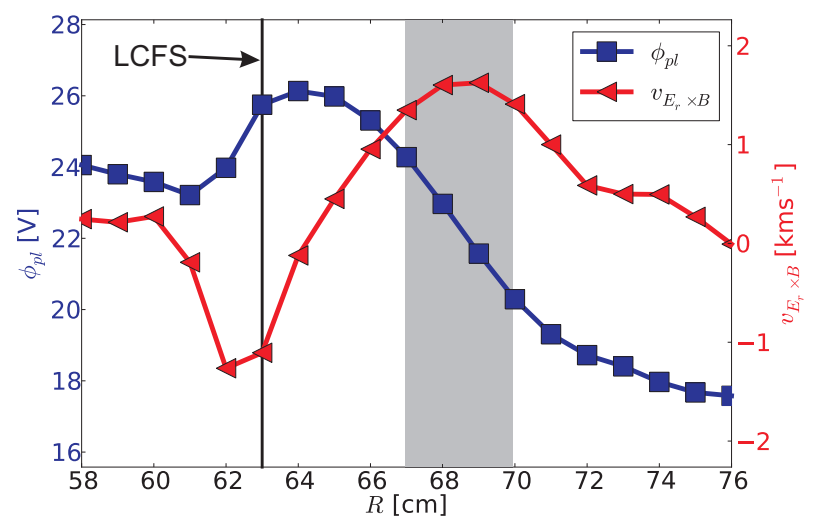

Figure 5. Typical TJ-K radial plasma potential profile for a He discharge with the corresponding $E_{\mathrm{r}} \times B$ drift in the poloidal direction.

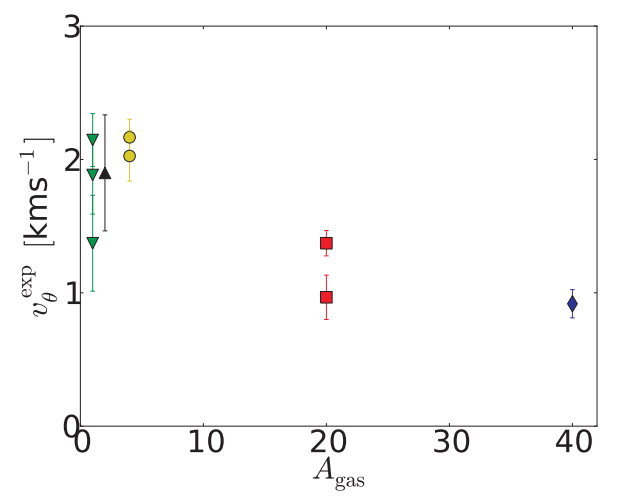

Figure 6. Poloidal blob velocity as a function of the mass number of the neutral gas, $A_{\text {gas }}$.

a typical radial plasma potential profile, along with the corresponding radial electric field. In the SOL the gradient in the plasma potential is negative, leading to a background $E \times B$ flow in the positive poloidal direction (ion diamagnetic direction). The grey shaded region indicates the radial range in which the blob velocity is averaged.

The analytical model [20 22] on which the equations in section 1 are based, has previously been found to compare well to experimental radial blob velocities in TJ-K in the inertial regime [13]. In this version of the model the radial blob velocity is related to the blob size $\delta_{\mathrm{b}}$, the approximated field line curvature $1 / R$, and the blob-background density ratio $\hat{n}=\tilde{n} / n$, via $v_{\mathrm{r}}=\sqrt{2 \delta_{\mathrm{b}} / R} c_{\mathrm{s}} \hat{n}[13,23]$. The model was extended in section 1 in order to incorporate the poloidal velocity component, $v_{\theta, \mathrm{b}}$.

CoM coordinates were used to extract local parameters for input into equation 

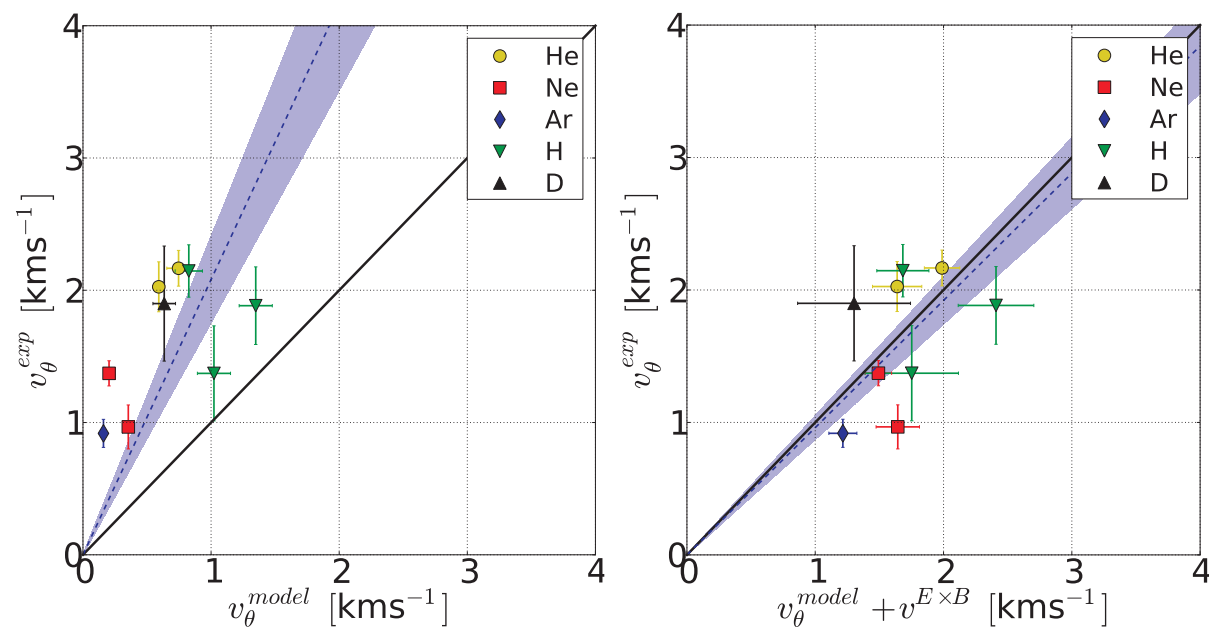

Figure 7. Experimental poloidal blob velocities compared to predictions from the analytical model with (right) and without (left) the addition of background poloidal $E \times B$ flow speeds.

7, namely the local background $T_{\mathrm{e}}$ and $n_{\mathrm{e}}$ measurements as well as the geodesic curvature. The temperature was determined from the difference between floating and plasma potentials, as previously done at TJ-K [28]. The geodesic curvature was obtained using an existing field line tracing code implemented with the TJ-K magnetic coil geometry. As an input to equation 7 , the curvature averaged along a field line bounded by the two poloidal limiters, $\left\langle\kappa_{\mathrm{g}}\right\rangle$, was used, as opposed to the local curvature. This accounts for the elongation of blobs parallel to the magnetic field [29]. In order to vary the parameter space, the gases H, D, He, Ne and Ar were used. A decreasing trend in the poloidal blob velocity at higher ion masses can be observed in figure 6, however the species $\mathrm{H}$ and $\mathrm{D}$ do not fit this trend.

Error bars for the poloidal velocities were calculated from the standard error on the conditionally averaged $I_{\mathrm{i} \text {,sat }}$ measurements, resulting from the averaging procedure. This error was used to determine a blob CoM error resulting in an error on the poloidal velocity. The error bars on the calculated model velocities were determined through propagating the errors on the experimental inputs to the model.

The left hand plot of figure 7 shows the experimental poloidal velocity components compared to the predictions of the analytical model in the inertial regime (as per equation 7). In this case the background plasma flow is not taken into account. The linear fit of the data, given by the blue dashed line, indicates a substantial deviation of the experimental data from the prediction of the analytical model. The slope gradient is $2.1 \pm 0.3$, where a slope of 1 means oneto-one correspondence between model and experiment. Thus in this case the model consistently underpredicts the absolute values of blob velocities, although the trend 
is still positive.

Blobs in TJ-K are propagating in a SOL plasma which has an equilibrium flow in the positive poloidal direction. In general measured poloidal velocities deduced from blob COM coordinates will not necessarily agree with a model incorporating only background $E \times B$ flow, i.e. $v_{\theta}^{\text {model }}+v^{\mathrm{E} \times \mathrm{B}}$, due to magnetic shear effects [30]. However, in the case of these experiments the affect of magnetic shear on the measured poloidal velocity can be neglected (see the next section).

The right hand plot of figure 7 shows the same experimental data, but with the addition of background $E \times B$ flow calculated from separate plasma potential measurements for each shot. The data points are scattered around the diagonal line representing one-to-one model-experiment correspondence and the slope of the linear fit of the data points is now $0.96 \pm 0.09$. The good agreement shows that the model can be used in TJ-K, with the addition of measured background flows to estimate the poloidal blob velocity, and in contrast to the radial velocity components calculated in [13], no cross-phase correct factor is required. Since the model is based on the geodesic curvature drive of blob polarisation, the result confirms the role of magnetic field line curvature in poloidal blob dynamics.

\subsection{The 3D structure of blobs}

Due to fast parallel transport, blobs are generally thought to be field-aligned structures. A consequence of this is that they should be influenced by magnetic field shear, becoming deformed in regions of high shear (e.g. [24, 25]). The purpose of this section is to investigate the $3 \mathrm{D}$ structure of blobs as they propagate through the SOL and to understand the effect of magnetic shear on this structure.

The three dimensional structure of blobs has been investigated using $\tilde{I}_{\mathrm{i} \text {,sat }}$ measurements in two different poloidal cross sections, separated by $60^{\circ}$ in the toroidal angle, $\phi$. At one toroidal position (port $\mathrm{O} 1, \phi=30^{\circ}$ ) fluctuations were measured using the $8 \times 8$ probe matrix, whilst at the other (port O6, $\phi=330^{\circ}$ ), the $2 \mathrm{D}$ scanning probe was used to take simultaneous measurements (see figure 1 for orientation). All fluctuations were conditionally averaged with a common reference signal, and the same trigger condition as described in section 3 was used.

In order to investigate the 3D structure over a substantial portion of the blob trajectory, the 64-probe matrix was positioned at 3 different points in the SOL, as illustrated in figure 8. These cover the blob trajectories typically observed with the particular experimental setup used for these experiments (see [13]). The same plasma conditions were obtained for each of the three shots, corresponding to the three distinct probe matrix positions.

To understand and quantify the degree to which blobs are aligned to the 


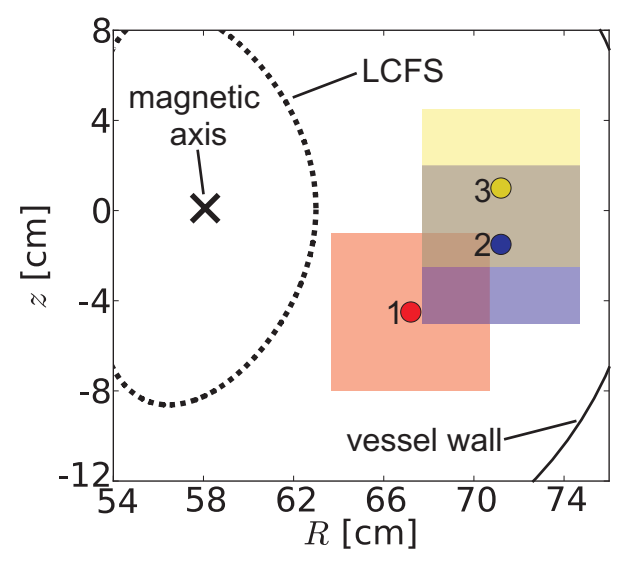

Figure 8. Measuring positions of the 64-probe matrix, labelled as positions 1,2 and 3 , shown in a poloidal cross-section of TJ-K.

magnetic field, blob structures occurring in the conditionally averaged probe matrix data were fitted with ellipses. The ellipse coordinates were then followed using a field line tracing code along the vacuum magnetic field lines to the poloidal plane containing the $2 \mathrm{D}$ scanning probe $(\mathrm{O} 1 \longmapsto \mathrm{O} 6)$. Figure 9 shows the conditionally averaged fluctuations at the two different toroidal positions, for a single time frame in the conditional average time series. The data was taken during He discharges at $B \approx 70 \mathrm{mT}$.

In order to characterise the pitch and tilt angles of the structures, two angles are defined for each ellipse, given by the subscripts 1 and 2. All angles are expressed in the interval $\theta \in[-\pi / 2, \pi / 2]$, with the line $z=0$ at $\theta=0$. In the case of the ellipse fitted to the conditionally averaged blob structure in the port O6 poloidal plane, $\alpha_{1}$ corresponds to the angle between the line connecting the magnetic axis to the blob centre of mass and the line $z=0$ (the horizontal). The angle $\alpha_{2}$ is that between the ellipse's major axis and the horizontal. The same applies to the ellipse fitted to the conditionally averaged data at port $\mathrm{O} 1(\beta)$, and the field-line-traced ellipse $(\gamma)$, as depicted in the figure. By calculating the angles $\alpha_{1}, \beta_{1}, \gamma_{1}$, the blob tilt can be measured relative to the field-aligned coordinate system as opposed to the Cartesian measuring coordinate system.

From plot 11 a) it can be seen how the blob CoMs determined at successive probe matrix positions have a relative poloidal shift. Since blobs in TJ-K propagate in the positive poloidal direction with this magnetic field orientation, the positions 1, 2 and 3 correspond to progressive locations along a typical blob trajectory.

The alignment of the blob structure to the magnetic field can be characterised 

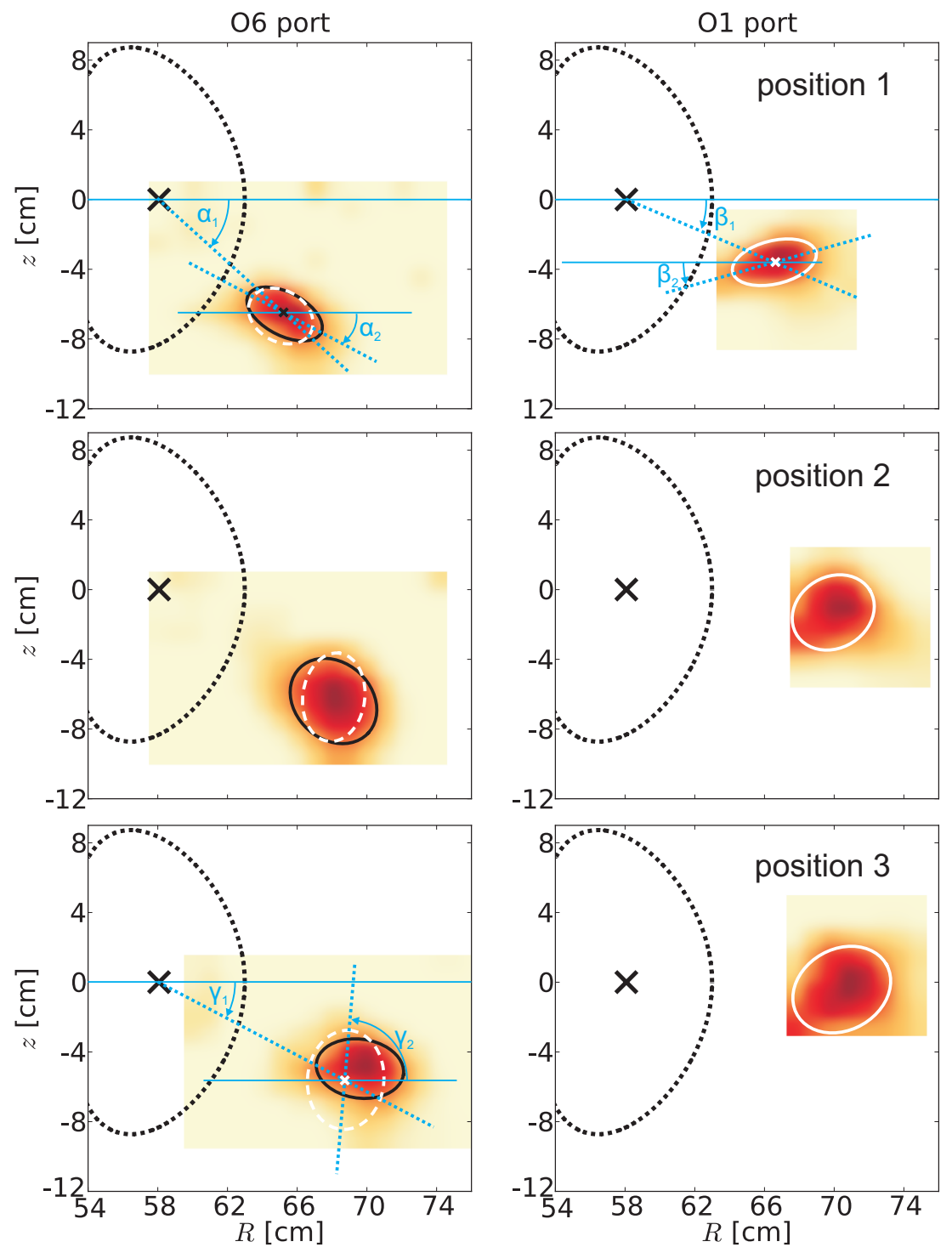

Figure 9. The conditional average of $I_{\mathrm{i}, \mathrm{sat}}$ fluctuations normalised by $\sigma_{\mathrm{I}_{\mathrm{i}, \mathrm{sat}}}$ at two toroidal positions, O6 (left) and O1 (right), separated by $60^{\circ}$, for the three probe matrix positions. In the left hand plots, the black ellipse is fitted to the conditionally averaged blob structure whilst the white dashedline ellipse corresponds to the ellipse obtained through field line tracing. In the right hand plots, a white ellipse is fitted to the conditionally averaged blob structure. The definitions of the characteristic ellipse angles $\alpha_{1,2}, \beta_{1,2}$ and $\gamma_{1,2}$ are depicted. 


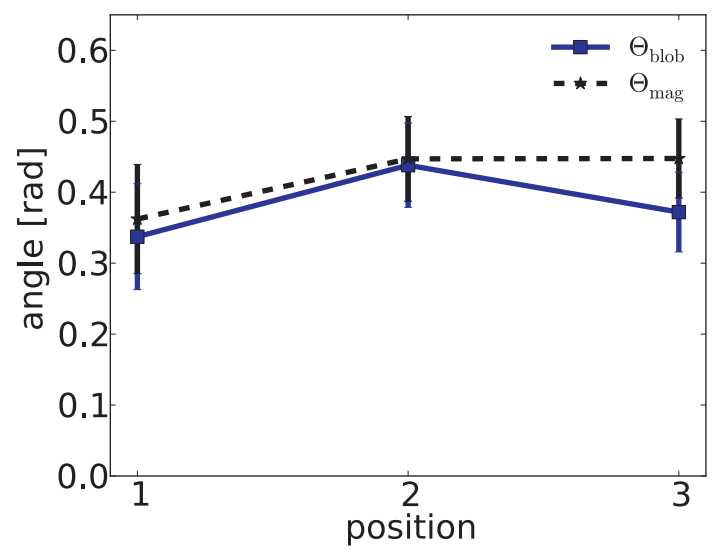

Figure 10. The pitch angle of the blob, $\Theta_{\text {blob }}$, and the magnetic field, $\Theta_{\text {mag }}$, as a function of the position of the probe matrix.

in part by the pitch angle of the filament, $\Theta_{\mathrm{blob}}$, compared to the pitch angle of the

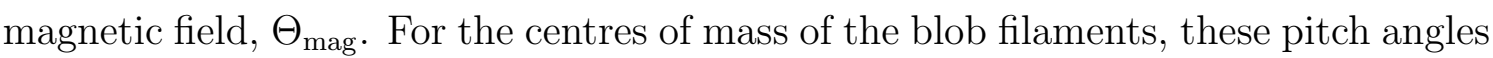
can be estimated from the information contained in the angles defined on figure 9 by calculating $\Theta_{\text {blob }}=\beta_{1}-\alpha_{1}$ and $\Theta_{\text {mag }}=\beta_{1}-\gamma_{1}$. From figure 10 it can be seen how in terms of the pitch angle the blob remains relatively well aligned at all 3 measuring positions, with a maximum misalignment of approximately $0.07 \mathrm{rad}$ at position 3 . This is not the case, however, for the blob tilt angle.

From plot 11b) it can be seen how the blob is initially field aligned in terms of its tilt angle, since $\alpha_{2}=\gamma_{2}$ to within the error bars at position 1. At positions 2 and 3 there is a significant difference between the two angles, quantifying the nonfield-alignment of the blob filament, which is evident from the contour plots in figure 9.

Finally, the angle $\alpha_{\mathrm{d}}$ is the difference, $\alpha_{\mathrm{d}}=\alpha_{2}-\alpha_{1}$ ( $\beta_{\mathrm{d}}$ is defined in the same way), which can be used to quantify the change in the relative tilt of a blob along its trajectory. The angular differences $\alpha_{\mathrm{d}}$ and $\beta_{\mathrm{d}}$ are shown in plot 11k). As a function of position, the angles $\alpha_{\mathrm{d}}$ and $\beta_{\mathrm{d}}$ do not change significantly within the error bars, indicating that the blob retains its original structure as it propagates through the SOL (note also that the change in the pitch angle defined by the ellipse CoM does not vary greatly between measuring positions). The same result has been previously observed in TJ-K for drift waves in the confined region [31], suggesting that it could be a property of coherent structures in TJ-K. In addition, a similar misalignment of blobs to the magnetic field has been observed in Alcator C-Mod [26, 32]. In TJ-K, the observation that blobs retain their original field aligned shape suggests that there 

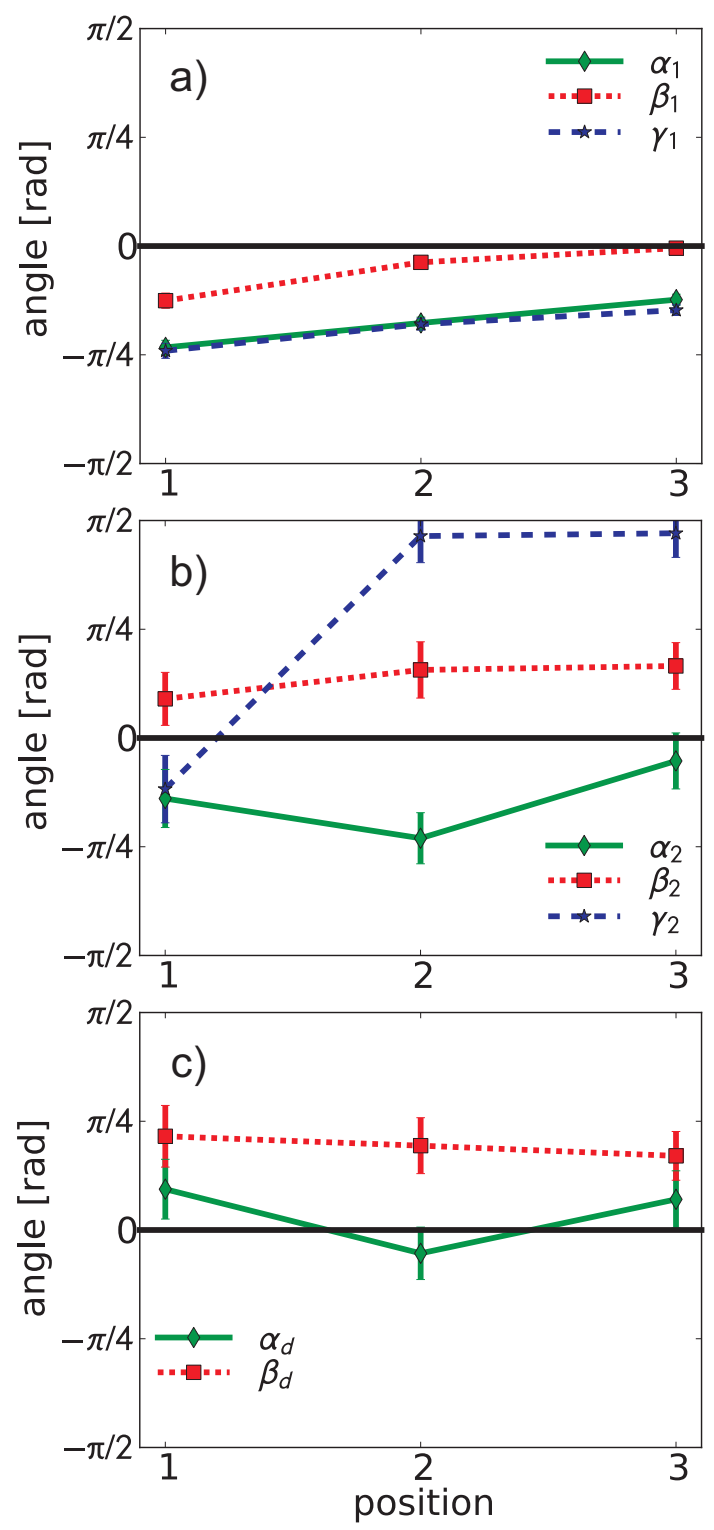

Figure 11. The ellipse angles $\alpha_{1,2, \mathrm{~d}}, \beta_{1,2, \mathrm{~d}}$ and $\gamma_{1,2}$ as a function of the 64-probe matrix position in the SOL.

is no further influence of magnetic field or $E \times B$ shear as the blob propagates, the reason for which is still unclear and requires further investigation.

\section{Conclusions}

Two experimental studies have been carried out on blobs at the TJ-K stellarator, into their poloidal velocity, and their 3D structure as they propagate through the SOL. The radial velocity component has already been investigated in TJ-K [13] and 
was found to agree well with an analytical blob model [20, 21, 23, 33], confirming the curvature drive of the radial velocity component, upon which the model relies. The same model can be used to express the poloidal velocity in terms of the geodesic curvature component, thereby allowing a comparison of poloidal blob velocities with the geodesic curvature drive. The predictions of the analytical model were found to deviate substantially from the experimentally measured velocities; when plotting the experimental velocity against the model prediction a linear fit gradient of $2.1 \pm 0.3$ was obtained, as opposed to a gradient of 1 for a one-to-one experimentmodel correspondence. The model does not, however, include the contribution of background $E \times B$ flows, which were deduced from direct measurements of the plasma potential using an emissive probe. With the simple addition of the background $E \times B$ flow to the model prediction, the experiment-model correspondence improves, with a linear fit of $0.96 \pm 0.09$. This confirms that the model can reasonably predict, quantitatively, the poloidal blob velocities in TJ-K, and since the model is based on a curvature drive, the geodesic curvature drive of poloidal blob velocity is also apparent.

Simultaneous measurements carried out in two poloidal cross sections, and at different positions in the SOL, have been used to probe the 3D structure of blob filaments in TJ-K. After they are generated, near the LCFS, filaments are aligned to the magnetic field. However, as they propagate through the SOL, their structure does not change according to the magnetic shear, but rather remains rigid, with the relative angular tilt of the filament staying as it was in the blob birth region. The reason for this misalignment to the magnetic field is not clear, however a similar effect has been observed for drift waves in the confined plasma of TJ-K [31], as well as blobs in Alcator C-Mod [26, 32]. The investigation of this effect is beyond the scope of this paper, and is left to further study.

\section{References}

[1] Zweben S J and Gould R W 1985 Nuclear fusion 25171

[2] D'Ippolito D A, Myra J R and Zweben S J 2011 Physics of Plasmas 18060501

[3] Lipschultz B et al. 2007 Nuclear Fusion 47 1189-1205

[4] Marandet Y et al. 2011 Nuclear Fusion 51083035

[5] Stroth U, Greiner F, Lechte C, Mahdizadeh N, Rahbarnia K and Ramisch M 2004 Physics of Plasmas 112558

[6] Rudakov D et al. 2005 Nuclear Fusion 45 1589-1599

[7] Grulke O, Terry J L, LaBombard B and Zweben S J 2006 Physics of Plasmas 13012306 
[8] Xu G et al. 2009 Nuclear Fusion 49092002

[9] Fuchert G, Birkenmeier G, Carralero D, Lunt T, Manz P, Müller H W, Nold B, Ramisch M, Rohde V and Stroth U 2014 Plasma Physics and Controlled Fusion 56125001

[10] Garcia O, Horacek J, Pitts R, Nielsen A, Fundamenski W, Naulin V and Rasmussen J J 2007 Nuclear Fusion 47 667-676

[11] Myra J, Davis W, D'Ippolito D, LaBombard B, Russell D, Terry J and Zweben S 2013 Nuclear Fusion 53073013

[12] Happel T, Greiner F, Mahdizadeh N, Nold B, Ramisch M and Stroth U 2009 Physical Review Letters 102255001

[13] Fuchert G, Birkenmeier G, Nold B, Ramisch M and Stroth U 2013 Plasma Physics and Controlled Fusion 55125002

[14] Tanaka K et al. 2006 Nuclear Fusion 46 110-122

[15] Banerjee S et al. 2012 Nuclear Fusion 52123016

[16] Poli F M, Ricci P, Fasoli A and Podest M 2008 Physics of Plasmas 15032104

[17] Carralero D et al. 2014 Nuclear Fusion 54123005

[18] Terry J, Zweben S, Grulke O, Greenwald M and LaBombard B 2005 Journal of Nuclear Materials 337-339 322-326

[19] Zweben S J et al. 2006 Physics of Plasmas 13056114

[20] D'Ippolito D A, Myra J R and Krasheninnikov S I 2002 Physics of Plasmas 9 222

[21] Garcia O E, Bian N H and Fundamenski W 2006 Physics of Plasmas 13082309

[22] Krasheninnikov S I, D'Ippolito D A and Myra J R 2008 Journal of Plasma Physics 74 679-717

[23] Theiler C, Furno I, Ricci P, Fasoli A, Labit B, Mller S H and Plyushchev G 2009 Physical Review Letters 103065001

[24] Myra J R, Russell D A and D'Ippolito D A 2006 Physics of Plasmas 13112502

[25] Antonsen T M, Drake J F, Guzdar P N, Hassam A B, Lau Y T, Liu C S and Novakovskii S V 1996 Physics of Plasmas 32221

[26] Grulke O, Terry J, Cziegler I, LaBombard B and Garcia O 2014 Nuclear Fusion 54043012

[27] Terry J, Zweben S, Umansky M, Cziegler I, Grulke O, LaBombard B and Stotler D 2009 Journal of Nuclear Materials 390-391 339-342

[28] Mahdizadeh N, Greiner F, Ramisch M, Stroth U, Guttenfelder W, Lechte C and Rahbarnia K 2005 Plasma Physics and Controlled Fusion 47 569-579 
[29] Fuchert G, Birkenmeier G, Ramisch M and Stroth U "Characterisation of the blob generation region and blobby transport in a stellarator" Plasma Physics and Controlled Fusion (this issue)

[30] Fedorczak N, Gunn J P, Pascal J Y, Ghendrih P, van Oost G, Monier-Garbet P and Tynan G R 2012 Physics of Plasmas 19072314

[31] Birkenmeier G, Ramisch M, Fuchert G, Köhn A, Nold B and Stroth U 2013 Plasma Physics and Controlled Fusion 55015003

[32] Zweben S J et al. 2013 Physics of Plasmas 20072503

[33] Theiler C, Furno I, Fasoli A, Ricci P, Labit B and Iraji D 2011 Physics of Plasmas 18055901 